\title{
12-Hour versus 24-Hour Creatinine Clearance in Critically Ill Pediatric Patients
}

\author{
SANDRA PONG, WINNIE SETO, MOHAMED ABDOLELL, ANGELA TROPE, KAREN WONG, \\ JOANN HERRIDGE, ELIZABETH HARVEY, AND BRIAN P. KAVANAGH \\ Department of Pharmacy [S.P., W.S., A.T., K.W.], Department of Critical Care Medicine [J.H., B.K.], \\ Department of Pediatrics [E.H.], The Hospital for Sick Children, Toronto, Ontario M5G 1X8, Canada; \\ Cancer Quality Council of Ontario Secretariat [M.A.], Cancer Care Ontario, Toronto, Ontario M5G 2L7, \\ Canada; Department of Public Health Sciences [M.A.], University of Toronto, Toronto, Ontario M5S 1A1, \\ Canada; Department of Pediatrics [E.H.], Interdepartmental Department of Critical Care Medicine
} [B.K.], University of Toronto, Toronto, Ontario M5S 1A1, Canada

\begin{abstract}
Measurement of renal function is important to optimize drug dosing in critically ill pediatric patients and to prevent doserelated toxicities caused by medications that are eliminated or metabolized by the kidney. In clinical practice, the 24 -h creatinine clearance $(\mathrm{CrCl})$ is used as a surrogate marker of renal function. However, a 24-h urine collection period delays the availability of the result and increases the potential for collection errors. This prospective, observational study was performed to determine whether a $12-\mathrm{h} \mathrm{CrCl}$ is comparable to the traditional 24- $\mathrm{h} \mathrm{CrCl}$ and to assess whether $\mathrm{CrCl}$ could be reliably predicted by the Schwartz equation, which mathematically estimates a child's GFR. A 24-h urine sample was collected in two 12-h aliquots from 60 catheterized critically ill children (age $2 \mathrm{~d}$ to $18 \mathrm{y}$ ). $\mathrm{CrCl}$ and Schwartz glomerular filtration rate (GFR) estimates were determined for each 12- and 24-h period. Agreement between 12- and 24-h $\mathrm{CrCl}$ and between $\mathrm{CrCl}$ and Schwartz GFR estimates was assessed using intraclass correlation coefficients
\end{abstract}

\section{ABSTRACT}

(ICCs). An ICC $\geq 0.8$ was considered to indicate excellent agreement. The ICC between the first $12-\mathrm{h} \mathrm{CrCl}$ and $24-\mathrm{h} \mathrm{CrCl}$ was 0.9605 . The ICC between the second $12-\mathrm{h} \mathrm{CrCl}$ and 24-h $\mathrm{CrCl}$ was 0.9602 . The ICC between the $24-\mathrm{h} \mathrm{CrCl}$ and Schwartz GFR was only 0.7046 . All comparisons of 12- and 24-h CrCl indicated excellent agreement. In summary, the Schwartz equation was not a reliable estimate of renal function in critically ill children, and a 12-h $\mathrm{CrCl}$ is just as accurate as the standard 24-h $\mathrm{CrCl}$ to assess renal function and guide drug dosing. (Pediatr Res 58: 83-88, 2005)
CrCl, creatinine clearance
GFR, glomerular filtration rate
ICC, intraclass correlation coefficient
ICU, intensive care unit
SCr, serum creatinine

Reliable and rapid assessment of renal function in critically ill pediatric patients is important to facilitate their appropriate treatment. If renal impairment is detected, then this could influence medical decisions regarding fluid balance and electrolyte replacement therapies as well as dosing of medications that are dependent on renal clearance. This is especially important for nephrotoxic medications to avoid toxicity resulting from drug accumulation and impaired drug elimination $(1,2)$. The glomerular filtration rate (GFR) is an accepted indicator of

Received May 28, 2004; accepted November 2, 2004.

Correspondence: Sandra Pong, The Hospital for Sick Children, Department of Pharmacy, 555 University Avenue, Toronto, Ontario M5G 1X8, Canada; e-mail: sandra.pong@sickkids.ca.

Supported by the Department of Critical Care Medicine and the Pharmacy Department Research and Education Fund.

Abstract previously published in Can J Hosp Pharm 2003;56(suppl):S48.

DOI: 10.1203/01.PDR.0000156225.93691.4F renal function, and several methods have been established to either measure or estimate the GFR (3-9).

Creatinine is an inert endogenous metabolic product of muscle metabolism that has a relatively constant production in the body and is present at fairly stable blood levels under most conditions (10). Because creatinine is filtered mainly through the glomerular capillary wall (8), a common method to estimate the GFR in both adults and children is to measure the 24-h creatinine clearance $(\mathrm{CrCl})$. This is calculated after analyzing the creatinine levels in blood and in a 24-h urine sample (Table 1, equation 1) (11).

For overcoming the need to collect urine over a prolonged period of time, equations have been developed to predict GFR or $\mathrm{CrCl}$ rapidly and easily, on the basis of a person's weight, height, age, and/or sex and assuming stable renal function and steady-state serum creatinine ( $\mathrm{SCr}$ ) levels $(5,10,12)$. One empirical formula that estimates GFR in the pediatric population is the Schwartz equation, which has been studied and used 
Table 1. Renal function assessment equations

Equation

Parameters

Equation 1 (11):

Creatinine Clearance $\left(\mathrm{mL} / \mathrm{min} / 1.73 \mathrm{~m}^{2}\right)$

$$
=\frac{\mathrm{U}_{\mathrm{cr}} \times \mathrm{V}_{\text {urine }}}{\mathrm{SCr}} \times \frac{1}{\mathrm{BSA}} \times 1.73 \mathrm{~m}^{2}
$$

Equation 2 (7):

$$
\mathrm{GFR}=0.55 \times \frac{\mathrm{ht}}{\mathrm{P}_{\mathrm{cr}}}
$$

Equation $3(6,15)$ :

$$
\mathrm{GFR}=\mathrm{k} \times \frac{\mathrm{ht}}{\mathrm{SCr}}
$$

- $\mathrm{U}_{\mathrm{Cr}}=$ urine creatinine $(\mu \mathrm{mol} / \mathrm{L})$

- $\mathrm{V}_{\text {urine }}=$ urine flow rate $(\mathrm{mL} / \mathrm{min})$

- $\mathrm{SCr}=$ serum creatinine $(\mu \mathrm{mol} / \mathrm{L})$; when midpoint values are not available, use average serum creatinine values from start and end of collection period

- $\mathrm{BSA}=$ body surface area $\left(\mathrm{m}^{2}\right)$

- $\mathrm{GFR}=$ glomerular filtration rate $\left(\mathrm{mL} / \mathrm{min} / 1.73 \mathrm{~m}^{2}\right)$

- ht $=$ height in $\mathrm{cm}$

- $\mathrm{P}_{\mathrm{cr}}=$ plasma creatinine concentration $(\mathrm{mg} / \mathrm{dL})$

- GFR = glomerular filtration rate $\left(\mathrm{mL} / \mathrm{min} / 1.73 \mathrm{~m}^{2}\right)$
- $\mathrm{ht}=$ height in $\mathrm{cm}$
- $\mathrm{SCr}=$ serum creatinine concentration $(\mu \mathrm{mol} / \mathrm{L})$
- $\mathrm{k}=$ age and sex dependent
Group
Low birth weight infants
Full-term infants $\leq 1$ y
Children 2-12 y
Females 13-21 y
Males 13-21 y

extensively in pediatric practice $(6,7,13,14)$. It was first derived in 1976 (Table 1, equation 2) and was based on the statistical analysis of height, plasma, and urine creatinine data in 186 noncritically ill children, ranging in age from 6 mo to $20 \mathrm{y}$ $(6,7)$. The Schwartz equation has since undergone several revisions. The Hospital for Sick Children has adopted a revised age- and sex-adjusted Schwartz equation (Table 1, equation 3) $(6,15)$ to estimate GFR in pediatric patients.

The assessment of renal function presents a unique challenge in critically ill pediatric patients because of varying degrees of renal function, altered body composition, abnormal fluid status, and fluctuations in hemodynamic stability. All of these alterations could compromise the achievement of steady-state $\mathrm{SCr}$ levels, invalidate assumptions of stable GFRs, and, therefore, preclude the use of empiric equations to accurately assess renal function $(8,12)$.

At present, the 24-h urine collection remains the standard period used in $\mathrm{CrCl}$ measurements. However, a 24-h collection delays the availability of measurement results and potentially increases the chance for collection errors to occur. This has been addressed in adult intensive care unit (ICU) patients, in whom 2-h urine collections have been demonstrated to achieve comparable accuracy with 24-h collections (2). However, more heterogeneous physiologic alterations, as well as smaller plasma and urinary volumes, mandate specific validation of abbreviated tests in critically ill children.

To date, no studies in the critically ill pediatric population have assessed the agreement between $\mathrm{CrCl}$ derived from a 24-h urine collection and $\mathrm{CrCl}$ obtained from a 12-h collection, and no comparisons have been made between measured 12- or 24-h $\mathrm{CrCl}$ and Schwartz GFR estimations. To address these issues, we investigated whether $12-\mathrm{h} \mathrm{CrCl}$ tests were comparable to traditional 24-h tests. In addition, we assessed the validity of the conventional Schwartz equation in critically ill children with altered renal function.

\section{METHODS}

Patients. Patients who were admitted to the pediatric ICU at the Hospital for Sick Children with an indwelling urinary catheter and had steady-state $\mathrm{SCr}$ levels defined as two consecutive SCr levels $24 \mathrm{~h}$ apart differing by $\leq 20 \%$ were included in the study. Patients were excluded when they received any form of renal replacement therapy (e.g. dialysis) or when they were anuric.

Study design. This prospective, observational study was conducted to determine whether 12-h $\mathrm{CrCl}$ measurements were comparable to 24-h $\mathrm{CrCl}$ measurements and to assess the validity of the conventional Schwartz equation in critically ill pediatric patients. All patients in the ICU were screened for inclusion criteria daily, and potential patients' parents/guardians were approached for consent to enroll the patients into the study. Assent or consent was obtained from patients as appropriate, according to guidelines set by the Hospital for Sick Children Research Ethics Board. The study was approved by the Hospital for Sick Children Research Ethics Board.

Demographic and admission information, including age, sex, height, weight, and medical conditions, was recorded. Information regarding urine output, fluid balance, medications, routine blood tests, protein balance, and ventilation status was collected to characterize the study population.

All urine produced by each patient and drained via his or her urinary catheter over a 24-h period was collected in two bottles in two consecutive 12-h intervals. Each urine sample was sent to the biochemistry laboratory to determine the urine volume and creatinine level. The 24-h urine collection was obtained by pooling the two consecutive 12-h samples. The coefficient of variation for the determination of creatinine levels in the serum and the urine was in the range of 3-5\% (Z. Verjee, Hospital for Sick Children, personal communication, 2001).

Serum creatinine levels that were obtained from routine blood work closest to the beginning and the end of each 12-h urine collection period were averaged to obtain midpoint SCr levels for each 12-h collection period. The midpoint SCr levels for the 24-h period were obtained by averaging the $\mathrm{SCr}$ levels from routine blood work that was obtained at the beginning of the first 12-h period and at the end of the second 12-h period. For each patient, $\mathrm{CrCl}$ was calculated using Table 1, equation 1, and GFR for each 12- and 24-h collection period was estimated using the Schwartz equation (Table 1, equation 3 ). 
Table 2. Patient characteristics

\begin{tabular}{lc}
\hline No. of patients $(n)$ & $57 *$ \\
Median age (range) & 7 mo $(2 \mathrm{~d}$ to $18 \mathrm{y})$ \\
Male sex $(\%)$ & $34(59.6 \%)$ \\
Negative protein balance $(n)$ & 57 \\
Ventilated $(\%)$ & $54(94.7 \%)$ \\
Use of potential nephrotoxins $(\%)$ & $35(61.4 \%)$ \\
$\quad$ Diuretics & $27(47.4 \%)$ \\
Aminoglycosides & $16(28.1 \%)$ \\
Vancomycin & $6(10.6 \%)$ \\
Amphotericin (regular and lipid) & $3(5.3 \%)$ \\
Cyclosporine & $2(3.5 \%)$ \\
Tacrolimus & * Sixty patients were recruited; one patient was excluded for accidentally \\
discarded urine sample, and two patients were excluded for sample leakage.
\end{tabular}

Sample size. Using the method proposed by Donner and Eliasziw (16), we determined that a minimum sample size of 50 patients was required to attain a level of significance $(\alpha)$ of 0.05 and power of $0.8(\beta=0.2)$ in a reliability study.

Demographic data. Nominal data were described by their relative frequencies. Continuous or discrete data were summarized by descriptive statistics. Microsoft Excel 2000 was used to perform the data analysis of demographic variables.

Agreement analyses. The agreements between 12- and 24- $\mathrm{h} \mathrm{CrCl}$ and Schwartz GFR estimates were assessed using three methods: correlation analysis, Bland and Altman method, and intraclass correlation coefficient (ICC) analysis.

Correlation analysis. Correlation analysis was used to measure the strength of linear association between each pair of renal function test methods compared $(17,18)$. Microsoft Excel 2000 was used to plot the correlation graphs and calculate the correlation equations.

Measure of agreement. Bland and Altman graphs plotting the difference between each pair of renal function values against the mean of each pair of values were used to evaluate the agreement between each pair of methods compared. For further assessing agreement, the $95 \%$ limits of agreement were calculated as the mean difference of the pairs of values $\pm 2 \mathrm{SD}$ of the difference (17-19). Microsoft Excel 2000 was used to plot the Bland and Altman graphs and to calculate the $95 \%$ limits of agreement.

ICC analysis. The ICC is a reliability coefficient ranging between 0 and 1 , with values closer to 1 representing stronger reliability. ICC values were calculated to objectively assess reliability between each pair of renal function assessment methods in terms of consistency and conformity $(20-22)$. This method accounts for differences between and within subjects, and an ICC $\geq 0.8$ represents good agreement (23). SPSS 10.1 for Windows was used to determine single-measure ICCs for each pair of methods compared, using a parallel, one-way random effect model and assuming equal variance in each case.

\section{RESULTS}

Sixty patients were enrolled in the study after consent was obtained from their parents/guardians. Reasons for excluding patients who otherwise met inclusion criteria were discharge from the ICU or removal of catheters within $24 \mathrm{~h}$ of admission to the ICU. Of the 60 patients who were recruited into the study, three were excluded from the final data analysis. The reasons for exclusion were accidental discard of a urine sample (one patient) and significant urine leakage from the catheter into the diaper (two patients). The demographic characteristics of the study population are presented in Table 2 . The distribution of the patients' reasons for admission is presented in Fig. 1. The majority of patients were admitted to the ICU after cardiovascular surgery. The outcomes of the agreement analyses-correlation equations and correlation coefficients $(r)$, Bland and Altman 95\% limits of agreement, and ICCsbetween 12- and 24-h $\mathrm{CrCl}$ and between $\mathrm{CrCl}$ and Schwartz

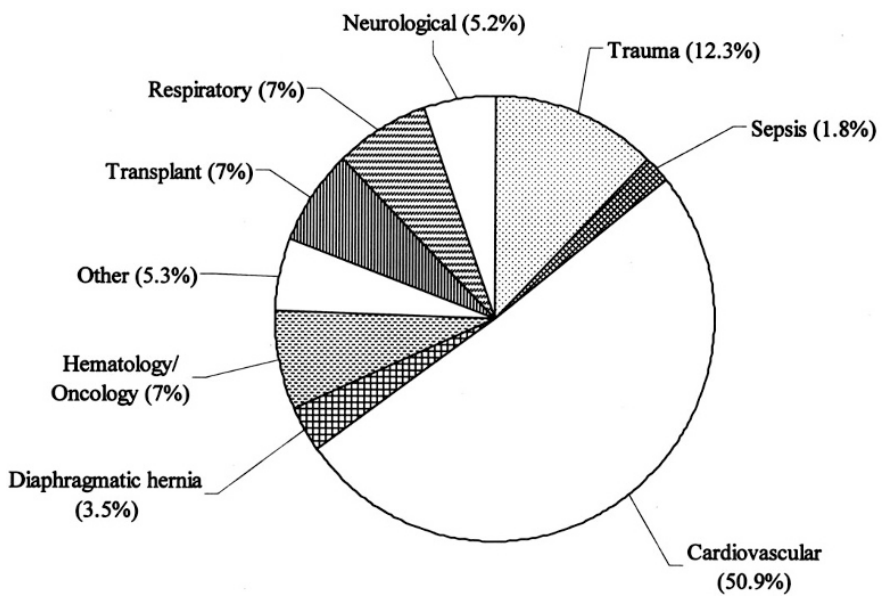

Figure 1. Reasons for admission to the ICU $(n=57)$.

GFR estimates for 12- and 24-h collection periods are summarized in Table 3.

Correlation analysis. The correlation coefficients $(r)$ were $>0.9$ when $\mathrm{CrCl}$ values for each 12-h period were plotted against 24-h $\mathrm{CrCl}$ values, suggesting a strong linear relationship between 12- and 24-h $\mathrm{CrCl}$ measurements. The correlation was also high when $\mathrm{CrCl}$ values from each 12-h period were plotted against each other. Linear relationships between measured $\mathrm{CrCl}$ and Schwartz GFR estimates were also evident with correlation coefficients ranging from 0.7654 to 0.8108 .

Bland and Altman method. The Bland and Altman technique was used to measure agreement by evaluating the difference between estimated GFR or measured $\mathrm{CrCl}$ for 12- or 24-h periods against the mean of each pair of methods being compared. Figure 2 provides a visual illustration of the agreement between measured 12- and $24-\mathrm{h} \mathrm{CrCl}$, assuming that the mean of the two methods compared was the best estimate of the true value.

The Bland and Altman values presented in Table 3 are the 95\% limits of agreement between each pair of methods compared. The mean difference indicated the bias of the methods being compared, and the SDs of the differences indicated the size of difference likely to arise between the

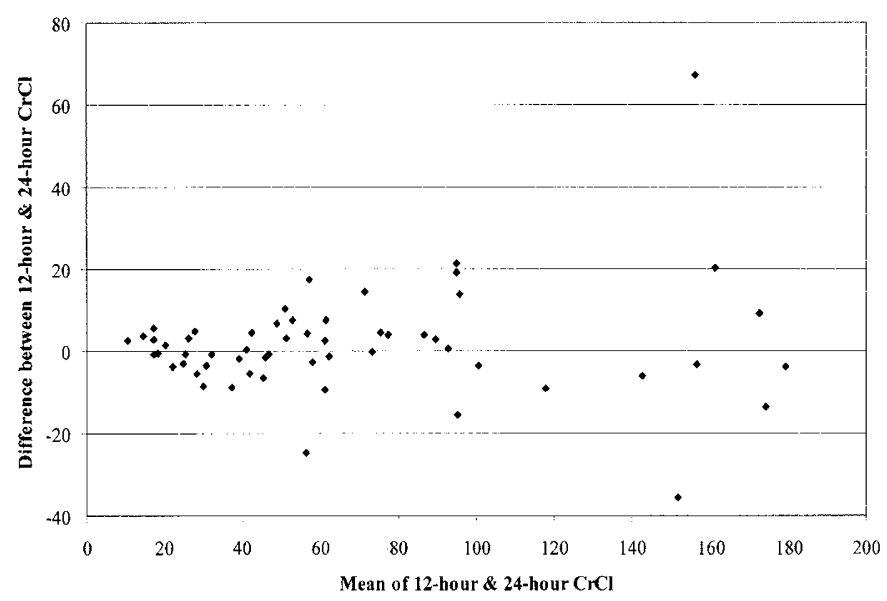

Figure 2. Bland and Altman plot of 12 -h (first 12 -h urine collection period) $\mathrm{CrCl}$ vs 24-h $\mathrm{CrCl}$. 
two methods (21). This approach enabled one to separate systematic error from random error but did not account for the possibility that each pair of methods compared may have agreed by chance.

ICC analysis. The ICC values presented in Table 3 for each pair of methods compared clearly show that there was good agreement between measured $12-$ and $24-\mathrm{h} \mathrm{CrCl}$ values because the ICC values in these comparisons all were $>0.8$. However, ICCs from comparisons of measured 12- or 24-h $\mathrm{CrCl}$ with Schwartz GFR estimates all had values of $<0.8$, indicating only moderate levels of agreement.

Subgroup analyses. Table 4 presents the Bland and Altman 95\% limits of agreement and the ICC values for 12- and 24-h $\mathrm{CrCl}$ comparisons in cardiac patients alone and in other medical-surgical patients. These subgroup analyses show comparable results with those of the pooled study population. Agreement between 12- and 24-h $\mathrm{CrCl}$ is good, regardless of whether the cardiac patients are analyzed alone or together with other medical-surgical patients who were admitted to the ICU.

\section{DISCUSSION}

In critically ill patients, non-steady-state creatinine kinetics limits the utility of equations that assess renal function (8,12,24-26). Studies by Kwong et al. (1) and Fong et al. (27) showed that the empiric Schwartz equation is not an accurate index of renal function in pediatric ICU patients. Because it is likely that medical decisions and drug dosing decisions that are affected by renal function will need to be addressed before the completion of prolonged urine collections and analyses, the clinical utility of endogenous $\mathrm{CrCl}$ may be improved and the inaccuracies associated with incomplete urine samples may be decreased by shortening the 24-h urine collection period to more convenient time intervals $(2,28-32)$.

Statistical analysis. The sole use of correlation analysis could not effectively determine the agreement between the various methods of renal function measurements assessed in the present study and answer the question of whether the various methods of measurement could be used interchangeably (20) because correlation analysis takes random error into account but is insensitive to systematic error (17-19). Therefore, the Bland and Altman method of agreement analysis was used to further analyze the data.

Bland and Altman graphs were used to investigate the relationships between measurement errors and true values. Divergence was observed in these graphs as the mean of each pair of methods compared increased, indicating that the measurement error increased with the size of the measurement. Although the Bland and Altman graphs visually depicted the agreement between each pair of methods, the degree of agreement could not be quantified and related to the range of the observations. Therefore, ICC analyses were performed to determine a single measure that would objectively assess reliability among each pair of methods compared.

Our study is the first to show that $12-\mathrm{h} \mathrm{CrCl}$ is in agreement with $24-\mathrm{h} \mathrm{CrCl}$ in critically ill pediatric patients. The ICC was always $>0.9$, regardless of whether the first 12 -h or the second $12-\mathrm{h} \mathrm{CrCl}$ was being compared with the $24-\mathrm{h} \mathrm{CrCl}$. The agreement between each 12-h $\mathrm{CrCl}$ was also high, with an ICC of 0.8553 . In contrast, the ICCs of the comparisons between measured $\mathrm{CrCl}$ and Schwartz GFR estimates for different urine

Table 3. Summary of statistical analyses

\begin{tabular}{|c|c|c|c|}
\hline Comparison & Correlation equation & $\begin{array}{c}\text { Bland and Altman } \\
\text { (difference } \pm 2 \mathrm{SD})\end{array}$ & $\mathrm{ICC}^{*}$ \\
\hline $12-\mathrm{h} \mathrm{CrCl}(\mathrm{A}) \dagger v s \quad 12-\mathrm{h} \mathrm{CrCl}(\mathrm{B}) \dagger$ & $\begin{aligned} \mathrm{y}= & 0.8345 \mathrm{x}+7.2181 \\
& (r=0.8567)\end{aligned}$ & $4.1 \pm 50.3$ & 0.8553 \\
\hline 12-h $\mathrm{CrCl}$ (A) vs 24-h $\mathrm{CrCl}$ & $\begin{array}{c}\mathrm{y}=0.9942 \mathrm{x}+1.893 \\
(r=0.9608)\end{array}$ & $1.5 \pm 26.4$ & 0.9605 \\
\hline 12-h CrCl (B) vs 24-h CrCl & $\begin{aligned} \mathrm{y}= & 0.9686 \mathrm{x}-0.4594 \\
& (r=0.9611)\end{aligned}$ & $-2.6 \pm 25.8$ & 0.9602 \\
\hline Schwartz 12-h (A) vs 12-h CrCl (A) & $\begin{aligned} \mathrm{y}= & 0.9038 \mathrm{x}+27.411 \\
& (r=0.8000)\end{aligned}$ & $-20.9 \pm 65.1$ & 0.7243 \\
\hline Schwartz 12-h (A) vs 12-h CrCl (B) & $\begin{aligned} \mathrm{y}= & 0.8877 \mathrm{x}+32.108 \\
& (r=0.7654)\end{aligned}$ & $-24.9 \pm 69.9$ & 0.6583 \\
\hline Schwartz 12-h (A) vs 24-h CrCl & $\begin{aligned} \mathrm{y}= & 0.9383 \mathrm{x}+26.478 \\
& (r=0.8026)\end{aligned}$ & $-22.4 \pm 64.4$ & 0.7105 \\
\hline Schwartz 12-h (B) vs 12-h CrCl (A) & $\begin{aligned} \mathrm{y}= & 0.9376 \mathrm{x}+27.496 \\
& (r=0.7967)\end{aligned}$ & $-23.2 \pm 67.9$ & 0.7041 \\
\hline Schwartz 12-h (B) vs 12-h CrCl (B) & $\begin{aligned} \mathrm{y}= & 0.9396 \mathrm{x}+31.168 \\
& (r=0.7778)\end{aligned}$ & $-27.3 \pm 70.6$ & 0.6509 \\
\hline Schwartz 12-h (B) vs 24-h CrCl & $\begin{aligned} \mathrm{y}= & 0.9813 \mathrm{x}+25.997 \\
& (r=0.8059)\end{aligned}$ & $-24.8 \pm 66.3$ & 0.6947 \\
\hline Schwartz 24-h vs 12-h CrCl (A) & $\begin{aligned} \mathrm{y}= & 0.9227 \mathrm{x}+27.852 \\
& (r=0.7955)\end{aligned}$ & $-22.6 \pm 67.3$ & 0.7079 \\
\hline Schwartz 24-h vs 12-h CrCl (B) & $\begin{aligned} \mathrm{y}= & 0.9241 \mathrm{x}+31.499 \\
& (r=0.7761)\end{aligned}$ & $-26.6 \pm 69.9$ & 0.6547 \\
\hline Schwartz 24-h vs 24-h CrCl & $\begin{aligned} \mathrm{y}= & 0.9731 \mathrm{x}+25.882 \\
& (r=0.8108)\end{aligned}$ & $-24.1 \pm 64.6$ & 0.7046 \\
\hline
\end{tabular}

* ICC $>0.8$ indicates good agreement.

$\dagger$ (A) denotes first 12-h urine collection period; (B) denotes second 12-h urine collection period. 
Table 4. Summary of subgroup analyses of cardiac versus medical-surgical patients

\begin{tabular}{ccc}
\hline Comparison & $\begin{array}{c}\text { Bland and Altman } \\
\text { (difference } \pm \text { 2 SD) }\end{array}$ & ICC* $^{*}$ \\
\hline Cardiovascular surgery patients & & \\
12-h CrCl (A) $\dagger$ vs 24-h CrCl & $-1.6 \pm 13.0$ & 0.9815 \\
12-h CrCl (B) $\dagger$ vs 24-h CrCl & $2.9 \pm 12.4$ & 0.9816 \\
Medical-surgical patients & & \\
12-h CrCl (A) vs 24-h CrCl & $-1.4 \pm 35.6$ & 0.9429 \\
12-h CrCl (B) vs 24-h CrCl & $3.0 \pm 34.8$ & 0.9424 \\
\hline
\end{tabular}

* ICC $>0.8$ indicates good agreement.

$\dagger$ (A) denotes first 12-h urine collection period; (B) denotes second 12-h urine collection period.

collection periods showed only moderate levels of agreement $($ ICC <0.8).

Potential limitations. In our study, the time at which the urine collections were started was not standardized. Several studies have suggested a circadian rhythmicity for $\mathrm{CrCl}$, which could introduce discrepancy in $\mathrm{CrCl}$ predictions depending on the time of the day. However, none have been able to substantiate such a phenomenon $(8,31)$. In the present study, urine samples were collected throughout a 24 -h period in two consecutive 12-h aliquots for each patient. Therefore, the urine collection period would have encompassed all, if any, circadian variations that would have occurred during the 24-h period.

Although $\mathrm{CrCl}$ was measured in all patients in the study, the measurement of $\mathrm{CrCl}$ has its own limitations. Because creatinine is secreted to some extent in the kidney, $\mathrm{CrCl}$ becomes an overestimation of the true renal function in patients who are renally compromised. Aside from using endogenous markers such as creatinine or urea and exogenous markers such as inulin or radioisotopes, another way to assess renal function is to measure the clearance of drugs such as aminoglycosides and vancomycin, which are primarily filtered through the kidneys and easily measured in urine and blood samples (33). By measuring the kinetic disposition of these medications in patients who received them as part of their drug therapy during the study, it may have been possible to compare their calculated $\mathrm{CrCl}$ and estimated Schwartz GFR values with their aminoglycoside or vancomycin pharmacokinetics. However, it was not the objective of the study to compare pharmacokinetics of drug dosing and renal function in patients but rather to use $\mathrm{CrCl}$ to help dosing of medications. Therapeutic drug monitoring was not performed intentionally for patients in the study, and not all patients were receiving aminoglycosides or vancomycin during the study period. Therefore, it was not possible to reliably extrapolate aminoglycoside or vancomycin kinetics in the context of the patients' $\mathrm{CrCl}$ measurements and Schwartz GFR estimates.

Cardiovascular surgery patients comprised $50.9 \%$ of the study population. This was a fair representation of the population of patients seen in the ICU at the Hospital for Sick Children. Despite the large proportion of cardiac patients compared with other patient groups, it was not likely that the cardiac patients influenced the results, because $12-\mathrm{h} \mathrm{CrCl}$ was compared with 24-h $\mathrm{CrCl}$ within each patient (cardiac patient or other) and not between different patient groups. Thus, each patient served as his or her own control. As presented in Table
4, separate Bland and Altman and ICC subgroup analyses of the agreement between 12- and 24-h $\mathrm{CrCl}$ in cardiac patients and other medical-surgical patients yielded comparable results to those of the pooled population.

\section{CONCLUSIONS}

Renal failure is an important determinant of morbidity and mortality. Children are an especially important group of critically ill patients because of their developmental potential and because many drug-dosing and toxicity profiles are not as well characterized in children as in adults. This study demonstrated that a 12-h urine collection period is just as accurate as the standard 24-h collection period to determine $\mathrm{CrCl}$. This finding has the potential to improve renal outcomes, drug use, and morbidity and mortality in critically ill children in a more timely manner.

Acknowledgments. We gratefully acknowledge the assistance and support of the nurses and physicians from the critical care unit. We also acknowledge both the Departments of Pharmacy and Critical Care Medicine at the Hospital for Sick Children for strong support and generous funding of this project.

\section{REFERENCES}

1. Kwong MB, Tong TK, Mickell JJ, Chan JC 1985 Lack of evidence that formuladerived creatinine clearance approximates glomerular filtration rate in pediatric intensive care population. Clin Nephrol 24:285-288

2. Wilson RF, Soullier G 1980 The validity of two-hour creatinine clearance studies in critically ill patients. Crit Care Med 8:281-284

3. Hernandez de Acevedo L, Johnson CE 1982 Estimation of creatinine clearance in children: comparison of six methods. Clin Pharm 1:158-161

4. Brochner-Mortensen J 1985 Current status on assessment and measurement of glomerular filtration rate. Clin Physiol 5:1-17

5. Papp CM, Nahata MC 1995 Prospective evaluation of ten methods for estimating creatinine clearance in children with varying degrees of renal dysfunction. J Clin Pharm Ther 20:67-73

6. Schwartz GJ, Brion LP, Spitzer A 1987 The use of plasma creatinine concentration for estimating glomerular filtration rate in infants, children, and adolescents. Pediatr Clin North Am 34:571-590

7. Schwartz GJ, Haycock GB, Edelmann CM Jr, Spitzer A 1976 A simple estimate of glomerular filtration rate in children derived from body length and plasma creatinine. Pediatrics 58:259-263

8. Robert S, Zarowitz BJ 1991 Is there a reliable index of glomerular filtration rate in critically ill patients? DICP 25:169-178

9. Cockcroft DW, Gault MH 1976 Prediction of creatinine clearance from serum creatinine. Nephron 16:31-41

10. Ravel R 1995 Clinical Laboratory Medicine-Clinical Application of Laboratory Data, 6th Ed. Mosby-Year Book, Toronto, pp 166-177

11. Atiyeh BA, Dabbagh SS, Gruskin AB 1996 Evaluation of renal function during childhood. Pediatr Rev 17:175-180

12. Kassirer JP 1971 Clinical evaluation of kidney function-glomerular function. N Engl J Med 285:385-389

13. Hellerstein S, Alon U, Warady BA 1992 Creatinine for estimation of glomerular filtration rate. Pediatr Nephrol 6:507-511

14. Springate JE, Christensen SL, Feld LG 1992 Serum creatinine level and renal function in children. Am J Dis Child 146:1232-1235

15. Roy R, Griffiths K (eds) 2003 The 2003-2004 Formulary of Drugs-The Hospital for Sick Children, 22nd Ed. The Graphic Centre HSC, Toronto, pp 77

16. Donner A, Eliasziw M 1987 Sample size requirements for reliability studies. Stat Med 6:441-448

17. Bland JM, Altman DG 1986 Statistical methods for assessing agreement between two methods of clinical measurement. Lancet 1:307-310

18. Streiner DL, Norman GR 1995 Health Measurement Scales: A Practical Guide to Their Development and Use, 2nd Ed. Oxford University Press, New York, pp $104-127$

19. Chatburn RL 1996 Evaluation of instrument error and method agreement. AANA J 64:261-268

20. Bland JM, Altman DG 1990 A note on the use of the intraclass correlation coefficient in the evaluation of agreement between two methods of measurement. Comput Biol Med 20:337-340 
21. Muller R, Buttner P 1994 A critical discussion of intraclass correlation coefficients. Stat Med 13:2465-2476

22. Portney LG, Watkins MP 2000 Foundation of Clinical Research: Applications of Practice, 2nd Ed. Appleton and Lange, Norwich, pp 557-586

23. Altman DG 1992 Practical Statistics for Medical Research. Chapman \& Hall, New York, pp 403-409

24. Robert S, Zarowitz BJ, Peterson EL, Dumler F 1993 Predictability of creatinine clearance estimates in critically ill patients. Crit Care Med 21:1487-1495

25. Martin C, Alaya M, Bras J, Saux P, Gouin F 1990 Assessment of creatinine clearance in intensive care patients. Crit Care Med 18:1224-1226

26. Seikaly MG, Browne R, Bajaj G, Arant BS Jr 1996 Limitations to body length/serum creatinine ratio as an estimate of glomerular filtration in children. Pediatr Nephro 10:709-711

27. Fong J, Johnston S, Valentino T, Notterman D 1995 Length/serum creatinine ratio does not predict measured creatinine clearance in critically ill children. Clin Pharmacol Ther 58:192-197
28. Richardson JA, Philbin PE 1971 The one-hour creatinine clearance rate in healthy men. JAMA 216:987-990

29. Markantonis SL, Agathokleous-Kioupaki E 1998 Can two-, four- or eight-hour urine collections after voluntary voiding be used instead of twenty-four-hour collections for the estimation of creatinine clearance in healthy subjects? Pharm World Sci 20:258 263

30. Sladen RN, Endo E, Harrison T 1987 Two-hour versus 22-hour creatinine clearance in critically ill patients. Anesthesiology 67:1013-1016

31. Baumann TJ, Staddon JE, Horst HM, Bivins BA 1987 Minimum urine collection periods for accurate determination of creatinine clearance in critically ill patients. Clin Pharm 6:393-398

32. Herget-Rosenthal S, Kribben A, Pietruck F, Ross B, Philipp T 1999 Two by two hour creatinine clearance-repeatable and valid. Clin Nephrol 51:348-354

33. Gambertoglio JG, Kearney BP 1999 Renal Disease. In: Anderson PO, Knoben JE, Troutman WG (eds) Handbook of Clinical Drug Data, 9th Ed. Appleton \& Lange, Stamford, pp 912-935 